\title{
Mucopolysaccharidosis and Adulthood: Genetics, Inheritance, and Reproductive Options
}

\author{
Alison Wilson ${ }^{1,2}$ Christine Lavery ${ }^{1, *}$ Fiona Stewart ${ }^{2}$ Sophie Thomas ${ }^{1}$ Deborah Cavell ${ }^{1}$ \\ Rebecca Brandon ${ }^{1}$ Elaine Murphy ${ }^{3}$ Gisella Wilcox ${ }^{4}$ Vikram Talaulikar ${ }^{5}$ \\ 1 Society of Mucopolysaccharide Diseases, Amersham, United Kingdom \\ 2 Northern Ireland Regional Genetics Service, Belfast City Hospital, \\ Belfast Health and Social Care Trust, Belfast, Northern Ireland \\ United Kingdom \\ ${ }^{3}$ Charles Dent Metabolic Unit, National Hospital for Neurology and \\ Neurosurgery, London, United Kingdom \\ ${ }^{4}$ Honorary Senior Lecturer, The Mark Hollan Metabolic Unit, Salford Royal NHS \\ Foundation Trust, University of Manchester, Manchester, United Kingdom \\ ${ }^{5}$ Reproductive Endocrinology and Assisted reproduction, University \\ College London Hospital, London, United Kingdom \\ Address for correspondence Alison Wilson, BSc, MSc, Genetic \\ Counsellor and Advocacy and Support Officer for MPS Society All \\ Ireland, Belfast City Hospital, Lisburn Road, Ireland \\ (e-mail: Alisona.Wilson@belfasttrust.hscni.net; \\ a.wilson@mpssociety.org.uk). \\ J Child Sci 2018;8:e138-e143.
}

\begin{abstract}
\section{Keywords}

- mucopolysaccharide

- genetic counseling

- inheritance

- prenatal

- preimplantation genetic diagnosis

- pregnancy

The mucopolysaccharide diseases are a group of heterogeneous, inherited conditions that are characterized by the deficiency of specific lysosomal enzymes. These lysosomal enzymes are responsible for the breakdown of glycosaminoglycans (GAGs), which are a key component of connective tissue. When lysosomal enzymes are deficient, the resultant build-up of GAGs impacts on cellular functioning. The resultant clinical manifestations are multisystemic, progressive, and life-limiting. Although each condition is characterized by its own constellation of symptoms; there is a great deal of heterogeneity and overlap in the clinical manifestations (both within and between mucopolysaccharidosis [MPS] subtypes). Increasing understanding of the genetic and biochemical basis of the MPS diseases has paved the way for a wave of exciting developments in their treatment and management. New treatments have changed the face of many of the MPS diseases; and as a result, management has moved beyond the pediatric clinic into the adult clinic. For the first time, individuals with MPS are living into adulthood with fewer limitations than the natural course of their disease would predict. It is essential that in this new age of MPS disease management, early diagnosis achieved and those at risk of having a child affected by one of these conditions are appropriately counseled in relation to their reproductive options. It is also important that individuals with MPS are counseled independently, at an appropriate age (or when they have capacity) about the basis of their disease and what this means for them. Here we discuss the diagnosis and inheritance of the MPS conditions; specifically focusing on genetic counseling requirements. We also discuss the outcomes of a research study, undertaken by the UK Mucopolysaccharide Disease Society, into the experiences of women with MPS who have had successful pregnancies.
\end{abstract}

\footnotetext{
deceased in December 2017
}

received

October 23, 2017

accepted after revision

May 10, 2018
Issue Theme Advances in

Mucopolysaccharidoses; Guest

Editors: Susanne G. Kircher, MD,

PhD, MBA, Adriana M. Montaño,

PhD, and Christina Lampe, MD.
Copyright @ 2018 Georg Thieme Verlag KG Stuttgart · New York
License terms

(®) $\Theta \circledast$

DOI https://doi.org/

10.1055/s-0038-1667347.

ISSN 2474-5871. 


\section{Introduction}

\section{Genetic and Biochemical Basis}

Establishing a diagnosis of a mucopolysaccharidoses (MPS) disease is not straightforward. There is a great deal of clinical overlap both within and between MPS subtypes. ${ }^{1-3}$ Consequently, correct diagnosis requires clinical expertise to determine the most appropriate choice of diagnostic tests. Timely diagnosis is of critical importance given the progressive nature of these conditions and the availability of disease specific treatments that have the potential to dramatically alter disease course and improve life expectancy, when delivered as early in the disease course as possible. ${ }^{4-7}$

In each of the mucopolysaccharide diseases, a specific enzyme, responsible for breaking down a specific glycosaminoglycan (GAG), is deficient. ${ }^{1}$ The enzyme deficiency is caused by alteration in a disease-causing gene. - Table 1 outlines the GAGs, enzymes, and genes associated with each of the 11 known MPS diseases. The molecular and biochemical basis is reviewed elsewhere. ${ }^{4,7,8}$

It is the combination of clinical suspicion, detection of specific GAG accumulation, determination of specific enzyme deficiency, and identification of a disease-causing mutation that definitively diagnoses an MPS disease.

Supporting individuals and families to understand the relationship between genes, enzymes, and GAGs provides an excellent basis for explaining the clinical manifestations, response to therapy, and the genetic transmission of the MPS diseases.

\section{The Pathway to Diagnosis}

Specialist laboratory testing is required in making an MPS diagnosis. ${ }^{4,5,7-12}$ In cases where there is an atypical presentation (e.g., an attenuated variant), the results of initial biochemical tests can be misleading. In these cases, it is essential that clinical evidence is carefully examined to determine the most appropriate line of testing. Specialist knowledge and expertise, alongside a close relationship between laboratory and clinical staff, is essential in identifying results that could lead to missed or misdiagnosis.

Where there is clinical suspicion of an MPS disease, the first stage in the diagnostic pathway is detection of the accumulation of storage product. For the MPS conditions, this is done by quantitative and qualitative analysis of urinary GAGs. ${ }^{10} \mathrm{~A}$ more specific diagnosis is then obtained by demonstrating a deficiency of a specific lysosomal enzyme. ${ }^{11-13}$ Clinical findings and the results of preliminary urine analysis are used in determining the most appropriate enzymes to assay. ${ }^{9}$ Enzymes are usually assayed in panels (multiplex assays ${ }^{12,13}$ ) based on preliminary test results and key clinical findings. Complete absence or very low activity of a specific enzyme, when compared with a reference range and assayed alongside positive and negative controls, ${ }^{14}$ typically provides a specific MPS diagnosis. ${ }^{9,12,13}$ As with preliminary biochemical testing, atypical cases will require case discussion.

The final step in obtaining a definitive diagnosis is identification of a disease-causing mutation(s) by genetic analysis. ${ }^{49}$ It is essential that this step is completed to ensure a definitive diagnosis and to facilitate risk clarification for the extended family. With each of the MPS diseases, the associated gene has been identified and isolated ${ }^{4,7,8}$; it is therefore possible to identify disease-causing mutations in most patients. When a known disease-causing mutation (one that has been previously described) is identified, this will provide a definitive diagnosis without further evaluation. However, with each of these very heterogeneous diseases, "private" family mutations are common and testing may identify sequence changes that have not been previously evaluated for pathogenic potential. In these cases, further studies may be required to elucidate pathogenicity.

The nature of the genetic mutation identified will have an impact on the genetic counseling of the affected individual and/or their relatives. Where a mutation has been well described, it may be possible to provide more detailed information about disease course and treatment outcomes.

Table 1 Summary of genetic and biochemical nature of mucopolysaccharide diseases

\begin{tabular}{|l|l|l|l|}
\hline MPS type & Gene location & Enzyme & Glycosaminoglycan \\
\hline MPS I (Hurler) & $4 \mathrm{p} 16.3$ & $\alpha$-lduronidase & HS and DS \\
\hline MPS II (Hunter) & Xq28 & Iduronate sulfatase & HS and DS \\
\hline MPS IIIA (Sanfilippo A) & $17 q 25.3$ & Heparan-N-sulfatase & HS \\
\hline MPS IIIB (Sanfilippo B) & $17 q 21.2$ & $\alpha$-N-acetylglucosaminidase & $\mathrm{HS}$ \\
\hline MPS IIIC (Sanfilippo C) & $8 \mathrm{p} 11.21$ & Acetyl-CoA- $\alpha$-glucosamine acetyltransferase & $\mathrm{HS}$ \\
\hline MPS IIID (Sanfilippo D) & $12 \mathrm{q} 14.3$ & N-acetylglucosamine 6-sulfatase & $\mathrm{HS}$ \\
\hline MPS IVA (Morquio A) & $16 q 24.3$ & Galactosamine-6-sulfate sulfatase & $\mathrm{CS}, \mathrm{KS}$ \\
\hline MPS IVB (Morquio B) & $3 \mathrm{p} 22.3$ & $\beta$-Galactosidase & $\mathrm{KS}$ \\
\hline MPS VI (Maroteaux-Lamy) & $5 \mathrm{q} 14.1$ & N-acetylglucosamine 4-sulfatase & $\mathrm{DS}$ \\
\hline MPS VII (Sly) & $7 \mathrm{q} 11.21$ & $\beta$-Clucuronidase & $\mathrm{CS}, \mathrm{HS}$, and DS \\
\hline MPS IX (Nantowicz) & $3 \mathrm{p} 21.31$ & Hyaluronidase 1 & Hyaluronan \\
\hline
\end{tabular}

Abbreviation: CS, chondroitin sulfate; DS, dermatan sulfate; HS, heparan sulfate; KS, keratan sulfate; MPS, mucopolysaccharidoses. 
While in cases where mutations have not been previously described, or where there is limited data, the information available to families may be less certain. Genetic counseling of families in cases where the pathogenic potential of the identified sequence changes is uncertain, requires expertise, and should be informed by multidisciplinary discussion.

\section{Next-Generation Sequencing}

As genetic testing capabilities advance and new technologies become more widely available, the use of next-generation sequencing (NGS) will increasingly result in the identification of MPS diseases in cases where clinical suspicion has not been raised. ${ }^{15}$ NGS involves the testing of large panels of genes. These panel tests are often used if there is a clinical presentation which is nonspecific (e.g., cardiomyopathy). Sequential testing in these situations can be long and costly. It is hoped that NGS will significantly reduce both cost and the time to diagnosis. In the case of the MPS diseases, this is of great advantage and will allow for quicker access to available therapies. It is also an advantage that these panel tests are increasingly becoming available in general medical settings. In cases where an MPS disease is identified as the result of panel testing, it is unlikely that families will have received pretest genetic counseling. This has a very significant impact on how families cope with the diagnosis of an inherited disease. Pretest counseling is known to have a positive impact on disease knowledge. ${ }^{16,17}$ It is, therefore, of critical importance that families are referred for genetic counseling and support following diagnosis.

\section{Inheritance and Genetic Counseling}

It is essential that families are supported in understanding the genetic basis of the diagnosis in their family. Not only does this understanding provide a framework on which to build information about the disease, its progression and the mechanisms of available treatments; but it is crucial in ensuring that families are aware of the risk of recurrence of the condition in the family. It also essential that those diagnosed with an MPS disease, when they have capacity, are supported in understanding the basis and reproductive implications of their condition.

Genetic counseling is the process of advising affected individuals and/or at-risk individuals about the basis, consequences, transmission, and treatment/management of a genetic disorder. ${ }^{18} \mathrm{~A}$ key aspect of genetic counseling is explanation about the inheritance pattern of the disorder in question. This information provides the basis on which the agenda of a genetic counseling session is set. Individuals with an MPS disease will inevitably be under the care of a wide range of medical professionals throughout their lifetimeeach with a specific remit and focus on a specific aspect of the disease in question. Genetic counseling is unique in its focus on the wider family rather than the affected individual in isolation. Crucially, genetic counseling is not limited to the provision of information about the disease. A genetic counselor will also address the emotional, familial, and societal implications of a genetic diagnosis in a family.
All but one of the MPS diseases are inherited in an autosomal recessive pattern. MPS type II (Hunter disease) is inherited in an X-linked pattern. For each mode of inheritance, it is important to take a detailed (at least three generation) family history to facilitate accurate risk estimate and effective risk communication. A genetic counselor will play a key role in supporting families to identify individuals who may benefit from genetic counseling and/or genetic testing.

\section{Autosomal Recessive Inheritance}

An individual will be affected by an autosomal recessive disease when they have a disease-causing mutation in both genes associated with the specific condition.

Both parents of an affected individual will be carriers of the condition and will have a 1 in 4 , or $25 \%$, risk of future pregnancies being affected by the same condition. Parents should be offered confirmatory carrier testing. It is essential that the parents of an affected individual are counseled prior testing about the expected results and the implications for future pregnancies. It is important to specifically highlight the potential for results which might raise questions regarding nonpaternity.

The siblings of an individual affected by an autosomal recessive condition are at a two in three chances of being carriers of the condition and should be offered the opportunity to discuss carrier testing at an appropriate age. Given that carrier status has no impact an individual's health, knowledge of carrier status is primarily sought when an individual is planning a family and considering their reproductive options. It is important that siblings are aware of the availability of genetic counseling as they approach reproductive maturity. When a sibling is identified as a carrier, it is then possible to counsel them about the risk to any future pregnancy. For some conditions, it is possible to offer carrier testing to the partner of a known carrier, even in the absence of a family history of the disorder. It is not possible to completely rule out that an individual is a carrier of a specific condition, but genetic testing can significantly reduce the risk, if a disease-causing mutation is not identified. In situations where this is not possible to test the partner of a known carrier, a more crude estimation of risk, based on approximate population frequency, is used to calculate the risk to future pregnancies. Given the rarity of the individual MPS diseases, it can be difficult to provide an accurate population frequency unless there are other known risk increasing factors such as consanguinity or population founder effects.

\section{X-Linked Inheritance}

Mucopolysaccharidosis Type II (Hunter disease) is X-linked recessive. $\mathrm{X}$-linked conditions are caused by disease-causing mutations in genes located on the X-chromosome. Hunter disease is associated with genetic alteration of the iduronate sulfatase gene.

Women who carry a disease-causing mutation on the iduronate sulfatase gene have a $50 \%$ chance of passing the gene on to their daughters, who will be carriers of the disease. Generally, females will have no health implications 
associated with being a carrier of Hunter disease; however, there have been a number of mutations carrying females who have developed symptoms of the condition as a result of either X-chromosomal abnormalities or nonrandom X-inactivation. ${ }^{19}$ These individuals have been reported as having symptoms similar to their male relatives. ${ }^{20-22}$

Equally there is a $50 \%$ risk of mutation carrying females passing the disease-causing mutation on to their sons. Sons of a carrier female who inherit the disease-causing mutation will, in all cases, be affected by Hunter disease.

Males with Hunter disease will pass on the diseasecausing gene to all of their daughters. There is no male-tomale transmission and therefore the sons of affected males will not be affected by the disease. Males with Hunter disease are not known to have any fertility issues associated with their condition and, where there is capacity and reproductive potential, males with Hunter disease should be counselled with regard to their reproductive options.

\section{Family Communication}

A genetic counselor will provide nondirective counseling about family communication. Families will be supported in identifying at-risk family members and planning communication with those who would benefit from genetic counseling advice. This is of particular importance in families with a diagnosis of Hunter disease where there is a possibility of carrier females of reproductive age being unaware of the risk and their reproductive options. For these women, their risk is not dependent on their partner. It is also of increased importance for families dealing with the diagnosis of a recessive disease where there is consanguinity or an increased carrier risk due to regional founder effects. In populations where there is an increased carrier risk, there is a much greater risk of transmission of the disease than in the general population. It is essential that this potentially increased risk is understood and that families are offered genetic counseling that is tailored to their specific circumstances. One example of a community with an increased carrier risk is the Irish Traveler community where there is a significantly increased risk of MPS type I (Hurler disease) due to the increased frequency of the W402X mutation in the community. ${ }^{23,24}$ In families where there is a recessive condition, but no other risk-increasing factors, the risk to a future pregnancy is likely to remain relatively low.

\section{Prenatal Diagnosis and Preimplantation Genetic Diagnosis}

For those individuals at a high risk of having a child affected by an MPS condition, it is possible to offer a range of reproductive options including both preconception testing (preimplantation genetic diagnosis [PGD]) and prenatal testing (free fetal DNA testing, chorionic villus sampling [CVS], or amniocentesis ). ${ }^{25,26}$ It is essential that those at risk of having a child affected by an MPS disease are counseling in relation to their reproductive options. This counseling is best delivered prior to pregnancy; however, in many cases women at a potentially increased risk will present to primary medical services early in pregnancy; these cases will require urgent action to facilitate genetic testing and clarification of risk.

The ethical issues surrounding prenatal testing have been widely discussed elsewhere 27,28 and the same challenges that have been described in more general reviews, such as guilt and conflict, also apply for the MPS diseases. It is essential that these issues are explored and that families are facilitated in finding their "own way" with regard to preconception and prenatal testing by the provision of clear and accurate information about the condition in question, options for testing, and the potential outcomes of testing. ${ }^{16}$

The first reproductive option outlined to any family will be the option of "doing nothing." Individuals at risk may wish to proceed with planning a family without any intervention or testing. This is perfectly acceptable and these families should be counseled in relation to the availability of testing postnatally. It is essential that individuals who chose not to test in pregnancy understand the value of early diagnosis.

For X-linked conditions, it is possible to first determine the sex of the fetus, using free fetal DNA testing. By taking a sample of blood from the mother in the early stages of pregnancy, from around 7 weeks, it is possible to detect fetal DNA which can be tested to determine if the fetus is male or female. In the case of Hunter disease, where females are very rarely affected, the results of this test may be useful in determining the appropriateness of further (more invasive) testing. In discussing this option with families, it is important that they understand that in very rare cases females can display symptoms of Hunter disease.

Disease-specific prenatal testing for MPS is invasive and involves ultrasound-guided biopsy of the placenta at 10 to 12 weeks (CVS) or sampling of the amniotic fluid at 16 to 17 weeks (amniocentesis). CVS is the preferred means of testing as it provides a result earlier in pregnancy. However, amniocentesis remains a very widely used technique. Samples from each method can be tested by enzyme or genetic analysis (when familial mutations are known). In the case of amniocentesis, cultured amniocytes can be used to detect GAG accumulation. It is usual for enzyme analysis to be performed in the first instance as this provides a rapid diagnosis and avoids the ethical considerations relating to the identification of gene carriers. The identification of a gene carrier through prenatal diagnosis brings with it a range of ethical considerations that should be presented and discussed by a genetic counselor. ${ }^{28}$

It is essential that, prior to prenatal testing, couples are supported in understanding the potential outcomes of testing. It is also important that couples consider what their response to a positive test (identification of an affected fetus) might be. Testing in the prenatal period comes with a great deal of emotion and families should be supported to consider their options before they are presented with a result. While some families will seek testing for the purposes of early diagnosis and the opportunity to commence treatment at birth, others may seek termination of pregnancy. Both of these paths come with unique moral, ethical, and emotional issues that should be explored fully with the support of a genetic counsellor. 
Families who do not feel that prenatal testing is an option for them may seek testing prior pregnancy. PGD is an assisted reproductive technology that involves in-vitro fertilization followed by genetic analysis of DNA from the resultant embryos for disease-causing mutation(s). The process of PGD relies upon the disease-causing mutation(s) in the parent(s) being identified so that these can be specifically tested for in the embryos. Following the testing of embryos, those that are unaffected are selected for implantation. In some cases, where there are no unaffected embryos available, carrier embryos will be implanted. The implantation of carrier embryos requires careful consideration given that a carrier will potentially have the same issues with regard to disease transmission when they reach reproductive maturity. Carrier embryos will only be implanted with the consent of the parents. It is important that families are counseled about the misdiagnosis rate associated with PGD which will be specific to their individual case.

\section{Pregnancy Study Outcomes}

The MPS Society has recently concluded a study into the needs and experiences of women with MPS diseases who have had successful pregnancies. The aim of this study was to, for the first time, interview women with MPS and related conditions who have had successful pregnancies to gain an insight into their needs and experiences during this time.

The study recruited six participants. Three MPS diseases were represented in the study cohort: MPS I (Hurler-Scheie), MPS I (Scheie), and MPS IV (Morquio). The cohort included both women treated by enzyme replacement therapy (ERT) and individuals who were naïve to treatment. The study involved semistructured telephone interviews which encompassed five core elements: general issues relating to the study participants and their underlying diagnosis, family history, issues surrounding the preconception and planning stages, pregnancy and birth, and postnatal care.

\section{Planning and Conception}

Participants' experiences of preconception counseling were mixed; with some receiving no preconception counseling at all while others spent time in detailed conversation with their metabolic consultant. The study identified that, although all participants demonstrated reasonable understanding of the genetic inheritance of their condition, only one participant received formal genetic counseling-this participant described a positive experience and felt "empowered" by the information that she had been provided with. Genetic counseling led to a clearer understanding of risk and the participant spoke at length about the benefit this aspect of planning had for her partner.

No participants in the study experienced any fertility problems despite all of the participants recalling that they had at some time been told that they would not be able to have children. One participant stated: “....my parents were told when I was little that I wouldn't live long enough to have children. So I didn't even think about it until I was married. Then I met my husband and panicked about our future. It would have been helpful to have had the conversation earlier."
In relation to preconception counseling, recommendation of this group is to incorporate preconception and genetic counseling into routine transitional care for MPS patients.

\section{Pregnancy and Birth}

In this part of the study, participants were interviewed in detail about their experience of each trimester of pregnancy. The interview included both medical and social questions; as well as providing an opportunity for participants to raise specific issues that were relevant to them.

Participants described having typical early pregnancy symptoms (including sickness, back pain, and fatigue). The key difference with these women was the level of anxiety about "what might happen" as their pregnancies progressed. Women reported concerns about how their bodies would cope with the additional pressures of pregnancy and uncertainty surrounding how their pregnancy should be managed. Participants described differing levels of follow-up, approaches to pain management, advice about medications (specifically the continued use of ERT during pregnancy), and advice about birth choices. These differing experiences left mothers-to-be feeling confused and often frustrated. A lack of interaction between specialties and lack of understanding of MPS was a key concern for the women interviewed. This lack of consistency was a great source of anxiety. One woman stated: "It was exciting to be pregnant! But it was scary not knowing what to expect. It was even scarier that my doctors didn't know either."

Exacerbation of common MPS issues such as orthopedic pain and respiratory stress was common problems for participants, particularly in the later stages of pregnancy.

Of the seven births covered by this study, there was one vaginal delivery at 29 weeks, four planned cesarean sections at $37,38,37$, and 39 weeks, and two emergency cesarean sections at 32 and 38 weeks.

The recommendation of the group is that the medical community develop peer-reviewed guidelines for the management of these complex pregnancies. There is a need for the introduction of a prepregnancy preparation clinic (a joint Metabolic/Obstetrics Clinic) for women with MPS diseases that should encompass a medication review, pain management discussion, pregnancy risks discussion, and preliminary birth planning. At this stage, women should be referred for baseline investigations (cardiac, respiratory, and spinal) and should receive genetic counseling. This should ensure that women feel informed and empowered at the start of their pregnancy and that their ongoing care is mapped out.

\section{Postnatal Care}

Participants described varying experiences of postnatal care and recovery. Some new mothers were able to return to normal activities soon after birth, while others required significant/intensive care and support to look after themselves and their new baby. Pain, fatigue, and reduced mobility (including the requirement for mobility aids) were the most limiting factors in the postnatal period. Although participants described excellent care and support by midwifery and nursing staff, there was a clear gap in MPSspecific knowledge. Again there were variations in advice 
relating to frequency of review appointments, the use of ERT (particularly while breastfeeding), and the availability of social support.

The recommendation of this group is that the medical community develop clear guidelines for the use of ERT in the postnatal/breastfeeding period. It is also essential that clear guidance is provided in relation to the frequency of review in the postnatal period. Specific training for midwives and community nursing staff should be developed.

All participants recounted how MPS impacted on being a new parent, with some finding the practicalities more challenging than they had anticipated. Fatigue, pain, and physical restrictions made day-to-day tasks more difficult to manage. Specific difficulties were discussed by participants related to manual handling, feeding, bathing, changing, managing equipment, and navigating their home with a new baby in their arms. Although some new mothers had excellent support from family, others were somewhat isolated during this period. Only one participant was offered social support from statutory agencies.

The recommendation of this group is that patient resources are developed in conjunction with experienced MPS parents to provide tailored practical advice. It is also recommended that information for healthcare professionals is developed to support a more in-depth understanding of the difficulties these new parents face. It is hoped that by improving awareness, new parents will be offered more tailored support packages.

\section{References}

1 Platt FM, Boland B, van der Spoel AC. The cell biology of disease: lysosomal storage disorders: the cellular impact of lysosomal dysfunction. J Cell Biol 2012;199(05):723-734

2 Wraith JE. The mucopolysaccharidoses: a clinical review and guide to management. Arch Dis Child 1995;72(03):263-267

3 Muenzer J. The mucopolysaccharidoses: a heterogeneous group of disorders with variable pediatric presentations. J Pediatr 2004; 144(5, Suppl):S27-S34

4 Hopwood JJ, Morris CP. The mucopolysaccharidoses. Diagnosis, molecular genetics and treatment. Mol Biol Med 1990;7(05): 381-404

5 Meikle PJ, Hopwood JJ. Lysosomal storage disorders: emerging therapeutic options require early diagnosis. Eur J Pediatr 2003; 162(1, Suppl 1):S34-S37

6 Beck M. Therapy for lysosomal storage disorders. IUBMB Life 2010;62(01):33-40

7 Clarke LA. The mucopolysaccharidoses: a success of molecular medicine. Expert Rev Mol Med 2008;18:10e1

8 Neufeld EF, Muenzer J. The mucopolysaccharidoses. In: Scriver CR, Beaudet AL, Sly WS, et al, eds. The Metabolic and Molecular Bases of Inherited Disease. New York: McGraw Hill; 2001: 3421-3452

9 Meikle PJ, Fietz MJ, Hopwood JJ. Diagnosis of lysosomal storage disorders: current techniques and future directions. Expert Rev Mol Diagn 2004;4(05):677-691
10 Hopwood JJ, Harrison JR. High-resolution electrophoresis of urinary glycosaminoglycans: an improved screening test for the mucopolysaccharidoses. Anal Biochem 1982;119(01):120-127

11 Kresse H, von Figura K, Klein U, Glössl J, Paschke E, Pohlmann R. Enzymic diagnosis of the genetic mucopolysaccharide storage disorders. Methods Enzymol 1982;83:559-572

12 Gelb MH, Turecek F, Scott CR, Chamoles NA. Direct multiplex assay of enzymes in dried blood spots by tandem mass spectrometry for the newborn screening of lysosomal storage disorders. J Inherit Metab Dis 2006;29(2-3):397-404

13 Zhang XK, Elbin CS, Turecek F, et al. Multiplex lysosomal enzyme activity assay on dried blood spots using tandem mass spectrometry. Methods Mol Biol 2010;603:339-350

14 Ruijter GJ, Boer M, Weykamp CW, et al. External quality assurance programme for enzymatic analysis of lysosomal storage diseases: a pilot study. J Inherit Metab Dis 2005;28(06):979-990

15 Mardis E. Next-Generations DNA Sequencing Methods. Ann Rev Genomics HumGenet 2008;9:387-402

16 Fonda Allen J, Stoll K, Bernhardt BA. Pre- and post-test genetic counseling for chromosomal and Mendelian disorders. Semin Perinatol 2016;40(01):44-55

17 Baldwin EE, Boudreault P, Fox M, Sinsheimer JS, Palmer CG. Effect of pre-test genetic counseling for deaf adults on knowledge of genetic testing. J Genet Couns 2012;21(02):256-272

18 Resta R, Biesecker BB, Bennett RL, et al; National Society of Genetic Counselors' Definition Task Force. A new definition of genetic counselling: national Society of genetic counsellors' task force report. J Genet Couns 2006;15(02):77-83

19 Tuschl K, Gal A, Paschke E, Kircher S, Bodamer OA. Mucopolysaccharidosis type II in females: case report and review of literature. Pediatr Neurol 2005;32(04):270-272

20 Winchester B, Young E, Geddes S, et al. Female twin with Hunter disease due to nonrandom inactivation of the X-chromosome: a consequence of twinning. Am J Med Genet 1992;44(06):834-838

21 Clarke JT, Greer WL, Strasberg PM, Pearce RD, Skomorowski MA, Ray PN. Hunter disease (mucopolysaccharidosis type II) associated with unbalanced inactivation of the $\mathrm{X}$ chromosomes in a karyotypically normal girl. Am J Hum Genet 1991;49(02): 289-297

22 Mossman J, Blunt S, Stephens R, Jones EE, Pembrey M. Hunter's disease in a girl: association with X:5 chromosomal translocation disrupting the Hunter gene. Arch Dis Child 1983;58(11):911-915

23 Murphy AM, Lambert D, Treacy EP, O'Meara A, Lynch SA. Incidence and prevalence of mucopolysaccharidosis type 1 in the Irish republic. Arch Dis Child 2009;94(01):52-54

24 Murphy AM. Disease frequency of inborn errors of metabolism in the Irish Traveller Community. Ulster Med J 2008;77(01):69

25 Lake BD, Young EP, Winchester BG. Prenatal diagnosis of lysosomal storage diseases. Brain Pathol 1998;8(01):133-149

26 Fowler DJ, Anderson G, Vellodi A, Malone M, Sebire NJ. Electron microscopy of chorionic villus samples for prenatal diagnosis of lysosomal storage disorders. Ultrastruct Pathol 2007;31(01): $15-21$

27 Benn PA, Chapman AR. Ethical challenges in providing noninvasive prenatal diagnosis. Curr Opin Obstet Gynecol 2010;22(02): 128-134

28 Powledge TM, Fletcher J. Guidelines for the ethical, social and legal issues in prenatal diagnosis. A report from the Genetics Research Group of the Hastings Center, Institute of Society, Ethics and the Life Sciences. N Engl J Med 1979;300(04):168-172 